\title{
Hoarseness after Section of a Cervical Vagus Nerve Schwannoma
}

\author{
Sung Hyun Noh', Ho Yeol Zhang ${ }^{1,2}$ \\ ${ }^{I}$ Department of Neurosurgery, National Health Insurance Service Ilsan Hospital, Goyang, \\ ${ }^{2}$ Department of Neurosurgery, Yonsei University College of Medicine, Seoul, Korea
}

\begin{abstract}
Schwannomas of the cervical vagus nerve are slow-growing asymptomatic solitary neck masses that rarely undergo malignant change. We report the case of a 41-year-old man who presented with a six-month history of a painless neck mass and paroxysmal coughing after palpation of the mass. Surgical excision of the mass was performed, and histopathological examination revealed that the mass was a schwannoma. The patient developed hoarseness after removal of the schwannoma, which originated from the fibers of the recurrent laryngeal nerve (RLN). We describe the relationship of the tumor and RLN and review the anatomy of the vagus nerve.
\end{abstract}

Key Words: Hoarseness $\cdot$ Neurilemmoma $\cdot$ Vagus nerve

\section{INTRODUCTION}

Schwannomas originating from the vagus nerve are uncommon benign nerve tumors, slow-growing masses, and asymptomatic lesions that are optimally managed via complete surgical resection. Radiologic studies play an important role in recognizing vagus nerve neoplasms. And the diagnosis is based on clinical examination findings and is confirmed via surgical pathological evaluation. Hoarseness is the most common complication of resection as a result of injury to the vagus nerve during surgery. Here, we report a patient undergoing hoarseness after removal of the schwannoma, which originated from the fibers of the recurrent laryngeal nerve (RLN). And we describe the relationship of the tumor and RLN and review the anatomy of the vagus nerve.

\section{CASE REPORT}

A 41-year-old man with no significant past medical history presented to our hospital with a six-month history of a palpable mass involving the left side of his neck. He complained that compressing or manipulating the neck mass provoked coughing. His physical examination revealed the presence of

Received: November 10, 2016, Revised: December 4, 2016

Accepted: December 30, 2016

Corresponding author: Ho Yeol Zhang, MD, PhD

Department of Neurosurgery, National Health Insurance Service Ilsan

Hospital, 100 \|lsan-ro, Ilsan-donggu, Goyang 10444, Korea

Tel: +82-31-900-0256, Fax: +82-31-900-0343

E-mail: hyzhang@nhimc.or.kr a soft, $5 \times 4 \mathrm{~cm}$ smooth mass in the left lower cervical region, and magnetic resonance imaging of the neck demonstrated a well-circumscribed mass exhibiting a high-intensity and heterogeneous signal on the left side of the neck. The mass was located between the common carotid artery and internal jugular vein (IJV) (Fig. 1). The location of the mass and its relationship to the vagus nerve and its branches are depicted in detail in Fig. 2.

Ultrasonography-guided fine needle aspiration biopsy of the mass was performed to obtain a pathologic diagnosis. However, no pathologic diagnosis was obtained. We therefore performed a tumor resection. With the patient under general anesthesia, a transverse cervical incision was made in the area involving the neck mass, and subcutaneous dissection was performed along the medial border of the sternocleidomastoid muscle. A $4 \times 3 \mathrm{~cm}$ yellowish-white, ovoid-shaped mass located
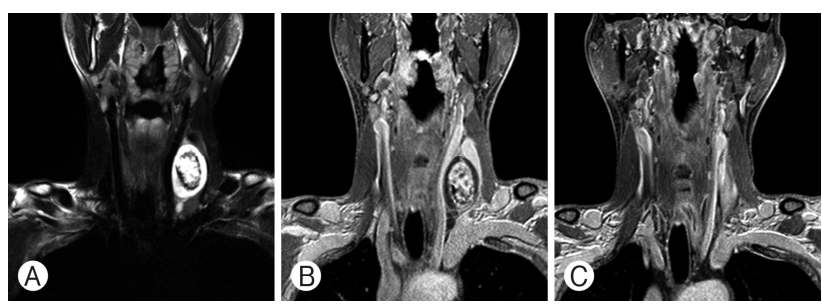

Fig. 1. (A) Coronal T2-weighted image (WI) demonstrating an ovoid mass in the left carotid sheath between the internal jugular vein (IJV) and common carotid artery. (B) Gadolinium-enhanced coronal Tl-WI demonstrating a well-circumscribed mass exhibiting a high-intensity and heterogeneous signal in the left carotid sheath between the IJV and common carotid artery. (C) Postoperative magnetic resonance imaging demonstrating that the tumor had been completely removed. 


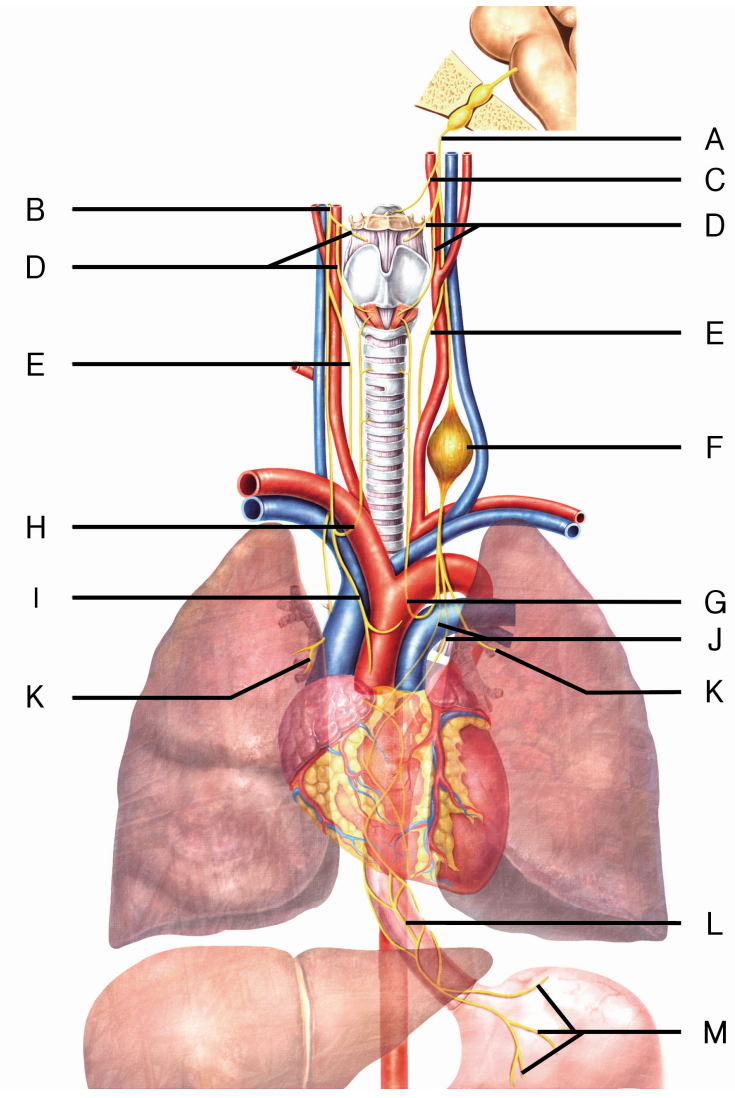

Fig. 2. Distribution of the vagus nerve. (A) left vagus nerve, $(B)$ right vagus nerve, (C) pharyngeal branches, (D) superior laryngeal nerve, (E) superior cervical cardiac branch, $(F)$ tumor, $(G)$ left recurrent laryngeal nerve $(\mathrm{RLN}),(\mathrm{H})$ right $\mathrm{RLN}$, (I) inferior cervical cardiac branch, $(\mathrm{J})$ cardiac plexus, $(\mathrm{K})$ pulmonary plexus, $(\mathrm{L})$ esophageal plexus, $(\mathrm{M})$ gastric plexus.

between the common carotid artery and IJV was observed. The tumor whose ends were each encircled by a vessel loop appeared to have arisen from the vagus nerve (Fig. 3A). A longitudinal incision was made along the tumor capsule to expose the well-encapsulated tumor before its enucleation and separation from the vagus nerve. Then, the proximal end of a vagus nerve fascicle was completely separated from the tumor, and complete tumor resection was performed using microsurgical instruments and techniques (Fig. 3B). However, the distal end of the above mentioned vagus nerve fascicle was adherent to the outer capsular surface of the tumor and therefore could not be separated clearly; as a result, we cut the distal end of the tumor with micro-scissors. The vagus nerve was preserved under surgical microscopic inspection, and a histopathological examination revealed that the tumor was a benign schwannoma.

The patient's cough resolved postoperatively; however, he developed hoarseness following surgery. An otolaryngologist conducted an examination of the larynx and diagnosed left
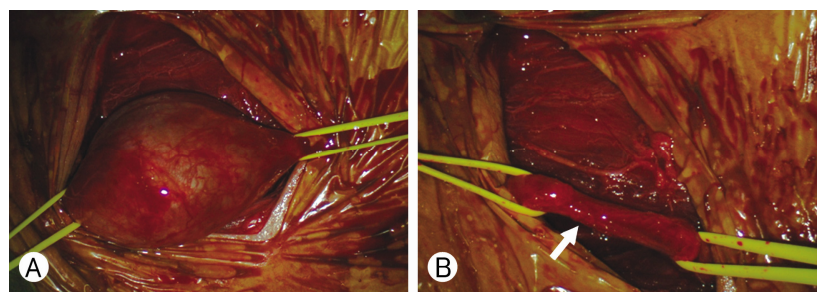

Fig. 3. (A) The proximal and distal ends of the tumor mass were encircled by vessel loops. (B) The tumor was completely removed, and the vagus nerve was preserved (arrow).
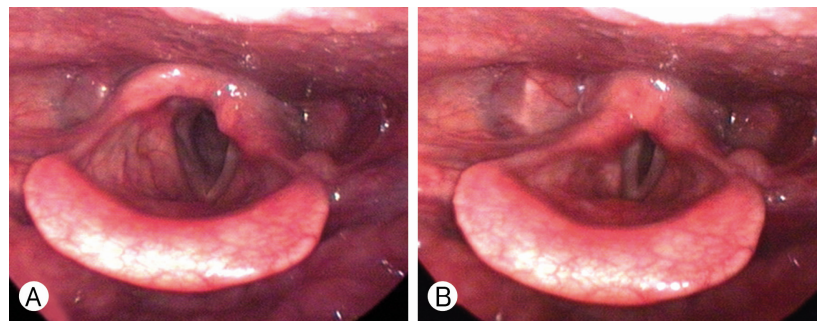

Fig. 4. (A) The vocal cords are in the open position during breathing. (B) The left vocal cord is partially closed during phonation as a result of unilateral vocal cord paralysis.

vocal cord palsy (Fig. 4), which was treated via the injection of hyaluronic acid (HA) directly into the left vocal cord. At follow-up at one year after surgery, the patient was well and exhibited no evidence of tumor recurrence. His vocal cord paralysis had improved but had not completely resolved.

\section{DISCUSSION}

Tumors of the parapharyngeal space are rare. The most common parapharyngeal tumors are salivary gland tumors, schwannomas, and paragangliomas. Neurilemomas (also known as schwannomas or neuromas) account for 55\% of parapharyngeal tumors ${ }^{8}$. Parapharyngeal schwannoma was first reported in 1933 by Figi $^{22}$. Approximately one-half of reported parapharyngeal schwannomas arise from the vagus nerve ${ }^{8)}$. Upon leaving the medulla oblongata between the pyramid and the inferior cerebellar peduncle, the vagus nerve spreads through the jugular foramen and then passes into the carotid sheath between the internal carotid artery and IJV, traveling down toward the neck, chest and abdomen, where it contributes to the innervation of the viscera (Fig. 2). The vagus nerve comprises cervical, thoracic, and abdominal segments. The cervical segment contains pharyngeal branches, as well as the superior laryngeal nerve, RLN, and cervical cardiac branches. The right RLN hooks underneath the right subclavian artery and then ascends toward the intrinsic muscles of the larynx, the majority of which it innervates. Similarly, the left RLN hooks under the arch of the aorta and then ascends toward 
the intrinsic muscles of the larynx, the majority of which it innervates. In our case, coughing occurred due to contraction of the intrinsic muscles of the larynx when the tumor was palpated because the mass arose from the fibers of the RLN, which branches off from the vagus nerve. Cavallaro et al. ${ }^{1}$ reported that hoarseness is the most common specific symptom due to vocal cord palsy, whereas the pathognomonic sign for vagal schwannoma is a paroxysmal cough during palpation of the mass due to vagal stimulation. Therefore, if patients cough when their tumors are palpated during preoperative examinations, physicians should consider the possibility that their tumors originate from the fibers of the RLN.

Gross total resection is the optimal treatment for vagus nerve schwannomas. The capsules should be removed gently and carefully to avoid injuring any remaining nerve fibers. The most common neurological complications of resection are associated with vagus nerve injury; thus, nerve-preservation techniques should be used. Any adhesions between the vagus nerve fibers and the tumor capsule require precise subcapsular dissection to preserve nerve function. Torossian et al. ${ }^{6}$ reviewed postoperative neurological outcomes in 15 patients with head-and-neck schwannomas who underwent enucleation with nerve preservation. Only two patients experienced tumor recurrences, which were attributed to a lack of microscopic dissection. Gibber et al. ${ }^{3)}$ used intraoperative nerve monitoring (INM) to aid the dissection of vagus nerve schwannomas. Specifically, they used an neural integrity monitor $\left(\mathrm{NIM}^{\circledR}\right)$ electromyographic (EMG) endotracheal tube (Medtronic, Minneapolis, MN, USA) to confirm that the nerve identified via dissection was the vagus nerve. RLN monitoring via an EMG endotracheal tube allows for intraoperative monitoring (IM) of nerve function and may decrease the risk of RLN injury in patients undergoing complex thyroid surgery or central neck lymph node dissection ${ }^{3}$. In our case, we operated more carefully if we used INM but were unsure whether to keep or cut the distal end of the tumor.

Hoarseness is reported by most patients following schwannoma resection, whereas vocal cord paralysis occurs in $85 \%$ of patients after tumor resection ${ }^{4)}$. Other common complications of schwannoma resection include pharyngolaryngeal anesthesia, aspiration and cranial nerve IX, XI and XII palsies, which may be transient or permanent.

Injectable materials, such as collagen-based products, autologous fat, HA, calcium hydroxylapatite, polymethyl methacrylate microspheres, and polyacrylamide hydrogels, can be used to treat vocal cord palsy ${ }^{7}$. HA, a nonsulfated glycosaminoglycan that is naturally present in the lamina propria of vocal folds as a principal component of the extracellular matrix, is another material suitable for treating vocal cord palsy ${ }^{7}$. A recent study in which 27 patients with unilateral vocal cord palsy underwent laryngoplasty with endoscopic injections of HA demonstrated that $87 \%$ of patients experienced subjective improvements in their voices postoperatively ${ }^{5)}$. In our case, the patient developed left vocal cord palsy postoperatively, for which HA was injected directly into the left vocal cord. The patient's vocal cord palsy subsequently improved but did not completely resolve.

\section{CONCLUSION}

Schwannomas originating from the vagus nerve are rare. Most affected patients present with slowly progressing neck swelling, without neurological deficits. If paroxysmal coughing is provoked by tumor palpation, physicians should suspect that the tumor involves the RLN branch of the vagus nerve. Vocal cord palsy is the most common complication of cervical vagus nerve schwannoma resection. IM is necessary during surgery to preserve the vagus nerve fascicles and RLN function.

\section{CONFLICTS OF INTEREST}

No potential conflict of interest relevant to this article was reported.

\section{ACKNOWLEDGEMENTS}

Prior presentation: The $5^{\text {th }}$ Korean Society of the Peripheral Nervous System Conference in 2013.

\section{REFERENCES}

1. Cavallaro G, Pattaro G, Iorio O, Avallone M, Silecchia G: A literature review on surgery for cervical vagal schwannomas. World J Surg Oncol 13:130, 2015

2. Figi FA: Solitary neurofibroma of the pharynx. Arch Otolaryngol 17:386-389, 1933

3. Gibber MJ, Zevallos JP, Urken ML: Enucleation of vagal nerve schwannoma using intraoperative nerve monitoring. Laryngoscope 122:790-792, 2012

4. Khafif A, Segev Y, Kaplan DM, Gil Z, Fliss DM: Surgical management of parapharyngeal space tumors: a 10-year review. Otolaryngol Head Neck Surg 132:401-406, 2005

5. Reiter R, Brosch S: Laryngoplasty with hyaluronic acid in patients with unilateral vocal fold paralysis. J Voice 26:785-791, 2012

6. Torossian JM, Beziat JL, Abou Chebel N, Devouassoux-Shisheboran M, Fischer G: Extracranial cephalic schwannomas: a series of 15 patients. J Craniofac Surg 10:389-394, 1999

7. Wen MH, Cheng PW, Liao LJ, Chou HW, Wang CT: Treatment outcomes of injection laryngoplasty using cross-linked porcine collagen and hyaluronic acid. Otolaryngol Head Neck Surg 149: 900-906, 2013

8. Yumoto E, Nakamura K, Mori T, Yanagihara N: Parapharyngeal vagal neurilemmoma extending to the jugular foramen. J Laryngol Otol 110:485-489, 1996 